\title{
Radiotherapy in prostate cancer treatment: results of the patterns of care study in Korea
}

\author{
Ah Ram Chang, MD, PhD ${ }^{1,3}$, Won Park, MD, PhD 2,3 \\ ${ }^{1}$ Department of Radiation Oncology, Soonchunhyang University Seoul Hospital, Soonchunhyang University College of \\ Medicine, Seoul; ${ }^{2}$ Department of Radiation Oncology, Samsung Medical Center, Sungkyunkwan University School of Medicine, \\ Seoul; ${ }^{3}$ Division for Urologic Cancer, Korean Radiation Oncology Group, Seoul, Korea
}

Purpose: The purpose of this study was to describe treatment patterns of radiotherapy (RT) for prostate cancer in Korea.

Materials and Methods: A questionnaire about radiation treatment technique and principles in 2013 was sent to 83 radiation oncologists and data from 57 hospitals were collected analyzed to find patterns of RT for prostate cancer patients in Korea.

Results: The number of patients with prostate cancer treated with definitive RT ranged from 1 to 72 per hospital in 2013. RT doses and target volumes increased according to risk groups but the range of radiation doses was wide (60 to $81.4 \mathrm{~Gy}$ ) and the fraction size was diverse (1.8 to 5 Gy). Intensity-modulated radiation therapy was used for definitive treatment in $93.8 \%$ of hospitals. Hormonal therapy was integrated with radiation for intermediate (63.2\%) and high risk patients (77.2\%). Adjuvant RT after radical prostatectomy was performed in 46 hospitals (80.7\%). Indications of adjuvant RT included positive resection margin, seminal vesicle invasion, and capsular invasion. The total dose for adjuvant RT ranged from 50 to 72 Gy in 24-39 fractions. Salvage RT was delivered with findings of consecutive elevations in prostate-specific antigen (PSA), PSA level over $0.2 \mathrm{ng} / \mathrm{mL}$, or clinical recurrence. The total radiation doses ranged from 50 to $80 \mathrm{~Gy}$ with a range of 1.8 to $2.5 \mathrm{~Gy}$ per fraction for salvage RT.

Conclusion: This nationwide patterns of care study suggests that variable radiation techniques and a diverse range of dose fractionation schemes are applied for prostate cancer treatment in Korea. Standard guidelines for RT in prostate cancer need to be developed.

Keywords: Prostate cancer, Radiotherapy, Patterns of care

\section{Introduction}

The patterns of care study (PCS) was developed in the United States by the American College of Radiology and was introduced to Korea to evaluate the current status of radiotherapy (RT) in 2002. The Korean Radiation Oncology Group (KROG) reported PCS results for RT in various kinds of cancers including breast, rectal, esophageal and cervical cancer [1-5].

A PCS for RT for prostate cancer was firstly reported in Korea in 2008. A questionnaire survey from 13 academic institutions was conducted, in which patterns of practice and principles of RT for prostate cancer treated in 2006 were inquired. The results of this study concluded that a nationwide Korean PCS is necessary as a baseline data to define RT guidelines for prostate cancer in Korea [6].

Received 21 September 2016, Revised 16 January 2017, Accepted 13 February 2017.

Correspondence: Won Park, MD, PhD, Department of Radiation Oncology, Samsung Medical Center, Sungkyunkwan University School of Medicine, 81 Irwon-ro, Gangnam-gu, Seoul 06351, Korea. Tel: +82-2-3410-2616, Fax: +82-2-3410-2619, E-mail: wonro.park@samsung.com

(c) This is an Open Access article distributed under the terms of the Creative Commons Attribution Non-Commercial License (http://creativecommons.org/ licenses/by-nc/4.0/) which permits unrestricted non-commercial use, distribution, and reproduction in any medium, provided the original work is properly cited.

www.e-roj.org 
For the first time, a nationwide survey on the use of RT in Korea was conducted by the Division for Urologic Cancer of the Korean Society for Radiation Oncology. The purpose of this study was to describe the treatment pattern of RT for prostate cancer in Korea.

\section{Materials and Methods}

We designed a questionnaire survey to describe treatment patterns of RT for curative aim for prostate cancer in Korea in 2013. This questionnaire included treatment modality, radiation field, prescribed dose, fractionation scheme, and followup and was sent by e-mail to 83 radiation oncologists in 83 different hospitals treating prostate cancer patients in Korea in 2014. The National Comprehensive Cancer Network (NCCN) risk grouping was used for risk stratification. It was defined as adjuvant RT even if there was a time delay after operation whenever RT was given without evidence of recurrences such as consecutive elevation of prostate-specific antigen (PSA) or clinical recurrence. The completed questionnaire forms were collected and analyzed. This study was conducted under the authorization and cooperation of the KROG (KROG 14-13).

\section{Results}

Sixty radiation oncologists out of 83 responded (72.3\%) and data from 57 hospitals was collected and analyzed to find RT patterns for prostate cancer patients in Korea. Three hospitals did not treat any prostate cancer patients with RT in 2014.

\section{Demographics}

Twenty-two hospitals responded to the questionnaire about the prostate cancer patients demographics in 2013. The number of all prostate cancer patients who were treated with any treatment for curative aim was 2,517 ranged from 6 to 617 in 22 hospitals. Among them, the number of prostate cancer patients treated with RT was 404 (16.1\%) out of 2,517 patients.

\section{Diagnosis and staging work up}

Twelve core biopsies were performed in $79.6 \%$ of hospitals that answered the questionnaire, and tertiary histology was obtained in 28 hospitals (49.1\%) for diagnosis.

Before RT, transrectal ultrasonography (TRUS), chest CT, pelvic or prostate $\mathrm{CT}_{1}$ pelvic or prostate $\mathrm{MRI}$, bone scan, and PET/CT were performed in 52 (91.2\%), 7 (12.3\%), 29 (50.9\%), 55 (96.5\%), 54 (94.7\%), and 7 hospitals (12.3\%), respectively (multiple answers possible).

\section{Technical aspects}

Planning MRI was used in 14 hospitals (24.6\%), but MRI fusion was used for target delineation in 29 hospitals (50.9\%).

Intensity-modulated RT (IMRT) was performed in 93.8\% of hospitals. External immobilization was used in $86 \%$ of hospitals and it was accompanied by IMRT and a 2-dimensional or 3-dimensional conformal RT technique in 48 $(84.2 \%)$ and 38 (66.7\%) hospitals, respectively. Most commonly, the VacLok system was used for external immobilization; the lower body was immobilized in 22 hospitals (38.6\%), while ankle immobilization was used in 16 hospitals (28\%) (multiple answers possible). Internal immobilization was used in $77.2 \%$. Bladder control commonly done with the bladder filling technique (answered in 30 hospitals), was performed in $70.2 \%$ of hospitals for internal immobilization. Rectal control including enema, gas passing, and rectal ballooning was used in $42.1 \%$ of responses.

The simultaneous integral boost (SIB) technique was used in 24 hospitals (42.1\%). Nineteen hospitals indicated that the SIB technique was used for elective whole pelvic RT, while 18 hospitals used SIB for gross tumor boost.

Preventive pelvic lymph node irradiation was indicated for patients with seminal vesicle involvement in 37 hospitals (64.9\%) and high-risk patients in 33 hospitals (57.9\%). Cone down was performed in 44 hospitals (77.2\%), mostly for IMRT (in 39 hospitals). Cone down was indicated in 33 hospitals (43\%) when whole pelvis RT was performed, and the seminal vesicles were irradiated in 26 hospitals (34.2\%).

\section{Definitive RT}

Table 1 shows target volume delineation for definitive RT according to risk grouping. Thirty hospitals (48\%) delineated the prostate and seminal vesicles as a clinical target volume (CTV) volume in the intermediate risk group. High-risk patients typically had the whole pelvis delineated as CTV volume. A prostate cancer stage of T3a or higher was the indication for CTV delineation of the seminal vesicles in 36 hospitals (63\%). Planning target volume (PTV) ranged from $2 \mathrm{~mm}$ to $15 \mathrm{~mm}$, except that in the posterior margin, a range of $1-8 \mathrm{~mm}$ was added to the CTV. The most common margin was $5 \mathrm{~mm}$, except for a posterior margin of $3 \mathrm{~mm}$.

The most commonly prescribed radiation dose was $72 \mathrm{~Gy}$ in 36 fractions, 70 Gy in 28 fractions, and 70 Gy in 28 fractions for low-, intermediate-, and high-risk patients, respectively. The radiation dose for seminal vesicles ranged from $46 \mathrm{~Gy}$ 
Table 1. Target volume delineation for definitive RT according to NCCN risk grouping

\begin{tabular}{|c|c|c|c|c|}
\hline \multirow{2}{*}{ Target volume } & \multirow{2}{*}{ Delineated volume } & \multicolumn{3}{|c|}{ No. of institutions (\%) } \\
\hline & & Low risk group & Intermediate risk group & High risk group \\
\hline \multirow[t]{4}{*}{ GTV } & No GTV & $9(16)$ & $8(14)$ & $8(14)$ \\
\hline & Gross tumor & $5(9)$ & $4(7)$ & $4(7)$ \\
\hline & $P G$ & $38(66)$ & $16(28)$ & $7(18)$ \\
\hline & $P G+S V$ & $6(0)$ & $29(51)$ & $38(67)$ \\
\hline \multirow[t]{6}{*}{ CTV } & No CTV $^{\mathrm{a})}$ & $22(29)$ & $10(16)$ & $30(33)$ \\
\hline & $P G$ & $31(40)$ & $6(10)$ & $0(0)$ \\
\hline & $P G+S V$ & $21(27)$ & $30(48)$ & $21(23)$ \\
\hline & Mini-pelvis ${ }^{b)}$ & $2(3)$ & $6(10)$ & $5(5)$ \\
\hline & Whole pelvis & $1(1)$ & $10(16)$ & $36(39)$ \\
\hline & $P G$ + proximal SV & $0(0)$ & $1(2)$ & $0(0)$ \\
\hline
\end{tabular}

The physicians selected multiple answers.

RT, radiotherapy; NCCN, National Comprehensive Cancer Network; GTV, gross tumor volume; CTV, clinical target volume; PG, prostate gland; SV, seminal vesicles.

a)'No CTV' means CTV is equal to GTV and only GTV was delineated. ${ }^{b}$ Mini-pelvis includes regional lymph nodes below the bottom of the sacroiliac joints.

Table 2. Details of the hormonal therapy combined with definitive RT according to NCCN risk grouping $(n=57)$

\begin{tabular}{llcc}
\hline \multirow{2}{*}{ Treatment } & \multicolumn{3}{c}{ No. of institutions (\%) } \\
\cline { 2 - 4 } & $\begin{array}{c}\text { Low risk } \\
\text { group }\end{array}$ & $\begin{array}{c}\text { Intermediate } \\
\text { risk group }\end{array}$ & $\begin{array}{c}\text { High risk } \\
\text { group }\end{array}$ \\
\hline Hormonal Tx & $14(25)$ & $36(63)$ & $44(77)$ \\
Yes & $43(75)$ & $21(37)$ & $13(23)$ \\
No & & & \\
Duration (mo) & $1(7)$ & $3(8)$ & $10(22)$ \\
$24-36$ & $1(7)$ & $0(0)$ & $0(0)$ \\
3 & $1(7)$ & $6(16)$ & $4^{\text {a) }}(9)$ \\
6 & $2(14)$ & $6(16)$ & $7(16)$ \\
Before recurrence & $9(64)$ & $22(59)$ & $24(53)$ \\
Not defined & & & \\
Timing & $0(0)$ & $4(19)$ & $9(25)$ \\
2 mo before RT & $6(75)$ & $6(29)$ & $9(25)$ \\
$3-6$ mo before RT & $0(0)$ & $0(0)$ & $3(8)$ \\
6 mo before RT & $1(13)$ & $9(43)$ & $14(39)$ \\
Start with RT & $1(13)$ & $2(10)$ & $1(3)$ \\
After RT &
\end{tabular}

RT, radiotherapy; NCCN, National Comprehensive Cancer Network. ${ }^{\text {a) }} 6-24$ months.

to 78 Gy in $20-39$ fractions (most commonly 50 Gy in 25 fractions).

Hormonal therapy (HT) was given to patients in $87.2 \%$ of hospitals. Low-risk patients were treated with HT at 14 hospitals (25\%) while intermediate- and high-risk patients were given with HT at 36 (63\%) and 44 hospitals (77\%), respectively. The duration of HT was not defined in all risk groups, HT was given 2 months before RT, 3-6 months before $\mathrm{RT}$, or started with RT at similar rates in intermediate- and high-risk patients (Table 2).

\section{Adjuvant RT}

Postoperative RT was applied in 46 institutions (80.7\%) for prostate cancer patients (Fig. 1A). Indications for adjuvant $\mathrm{RT}$ is shown in Fig. 1B. In 20 hospitals, adjuvant RT was given after recovery of incontinence. In most cases, adjuvant $\mathrm{RT}$ was performed after recovery of incontinence (Fig. 1C).

Table 3 shows CTV delineation for postoperative RT according to risk grouping. The PTV ranged from $1 \mathrm{~mm}$ to 15 $\mathrm{mm}$, except the posterior margin ranged from $1 \mathrm{~mm}$ to $8 \mathrm{~mm}$ and was added to the CTV. The most common margin was 5 $\mathrm{mm}$, except for the posterior margin of $3 \mathrm{~mm}$ when IMRT was applied for adjuvant RT.

The most common prescription dose for adjuvant RT was 66 $\mathrm{Gy}$ in 33 fractions. The total dose ranged from 50 to $72 \mathrm{~Gy}$ in 24-39 fractions. Cone down was done in 28 hospitals (49.1\%) and was mostly done once. Only 4 hospitals (7.0\%) performed cone down twice. Indications for cone down were whole pelvic treatment, large initial field size, and positive resection margin in 26 (52.0\%), 12 (24.0\%), and 11 hospitals (22.0\%), respectively.

HT was given to patients in $56.1 \%$ of hospitals. Indications for hormonal treatment included high Gleason score, initial high PSA level, presence of extracapsular extension, seminal vesicle invasion, positive resection margin, and positive pelvic LN metastases, with similar frequency. The duration of HT was 
A

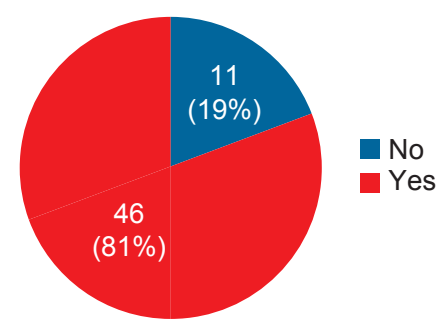

B

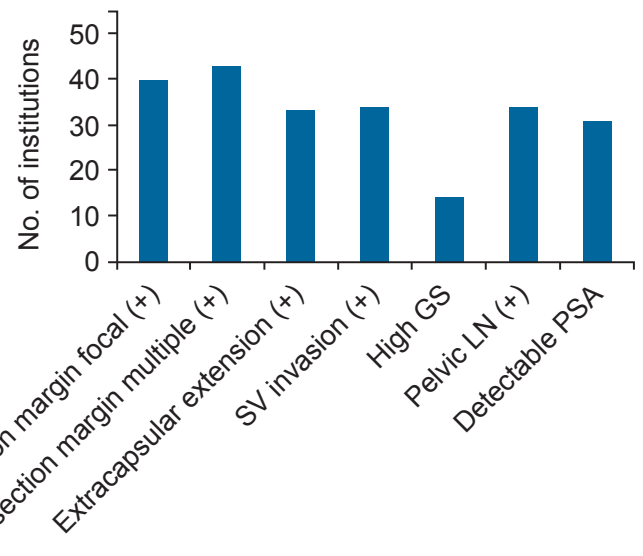

C

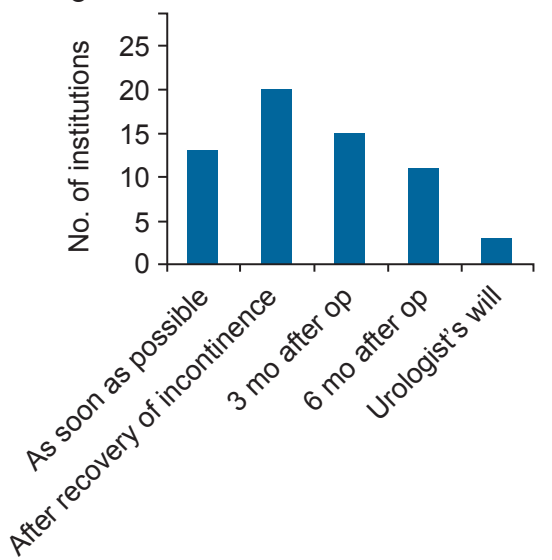

Fig. 1. (A) Postoperative radiotherapy (RT) in 2013. (B) Indications for postoperative RT in each institution. Multiple indications were allowed. (C) Timing of postoperative RT in each institutions. Multiple indications were allowed. SV, seminal vesicles; GS, Gleason score; LN, lymph node; PSA, prostate-specific antigen; op, operation.

Table 3. CTV delineation for postoperative RT according to NCCN risk grouping

\begin{tabular}{lcccc}
\hline & \multicolumn{3}{c}{ No. of institutions (\%) } \\
\cline { 2 - 5 } & Low risk group & Intermediate risk group & High risk group & Pelvic lymph node positive \\
\hline Surgical bed & $6(14)$ & $3(7)$ & $0(0)$ & $0(0)$ \\
Prostate bed & $15(35)$ & $2(4)$ & $1(2)$ & $1(2)$ \\
PG + partial SV bed & $8(19)$ & $13(29)$ & $4(9)$ & $1(2)$ \\
PG + SV bed & $10(23)$ & $15(33)$ & $10(23)$ & $0(0)$ \\
Mini-pelvis & $0(0)$ & $2(4)$ & $6(14)$ & $3(7)$ \\
Whole pelvis & $4(9)$ & $10(22)$ & $22(51)$ & $38(88)$ \\
\hline
\end{tabular}

The physicians selected multiple answers.

CTV, clinical target volume; RT, radiotherapy; NCCN, National Comprehensive Cancer Network; PG, prostate gland; SV, seminal vesicles.

not defined in 15 hospitals. The timing of HT also varied. HT was started with RT at12 hospitals, while HT was given 2-3 months before RT or started with RT at 7 hospitals.

\section{Salvage RT}

Salvage RT was applied in 46 institutions (80.7\%) for prostate cancer patients. Indications for salvage RT are shown in Fig. 2A. Consecutive PSA elevation was the most frequent indication for salvage RT in 35 hospitals (36.9\%). Salvage RT was indicated in 22 hospitals (23.2\%) when clinical recurrence was found, and 17 hospitals (18.1\%) had no guidelines.

Tumor extent, risk group before operation, and imaging results at recurrence determined the CTV (Fig. 2B). When there was no visible gross tumor, the prostate and partial seminal vesicle bed were commonly delineated as the CTV; while the prostate and partial seminal vesicle bed or the whole pelvis were commonly delineated. With pelvic LN metastases, the whole pelvis was designated as the CTV in 54 hospitals (Table 4).
The PTV margin ranged from $0 \mathrm{~mm}$ to $13 \mathrm{~mm}$, except that the posterior margin ranged from $0 \mathrm{~mm}$ to $8 \mathrm{~mm}$ and was added to the CTV. The most common margin was $5 \mathrm{~mm}$, except for the posterior margin of $3 \mathrm{~mm}$ when IMRT was applied for salvage RT.

A total dose of 66 Gy in 33 fractions was the most commonly prescribed dose for salvage $\mathrm{RT}$ in prostate cancer. A range of 50-80 Gy in 24-40 fractions was prescribed for salvage RT. Cone down was done in 39 hospitals (68.4\%) and was mostly done once. Indications for cone down were whole pelvis treatment, large initial field size, and the need to decrease rectal dose in 36 (63.2\%), 20 (35.1\%), and 1 hospital $(2 \%)$, respectively.

HT was given to patients in $77.2 \%$ of hospitals. Indications for hormonal treatment included high Gleason score, high PSA level at salvage, presence of gross tumor, and positive pelvic LN metastases. The duration of HT was not defined in 22 hospitals (64.8\%). HT was most commonly started with RT in 
Table 4. CTV delineation for salvage RT

\begin{tabular}{lccc}
\hline \multirow{2}{*}{ CTV } & \multicolumn{3}{c}{ No. of institutions (\%) } \\
\cline { 2 - 4 } & $\begin{array}{c}\text { No visible } \\
\text { gross tumor }\end{array}$ & $\begin{array}{c}\text { Visible gross } \\
\text { tumor }\end{array}$ & $\begin{array}{c}\text { Pelvic lymph } \\
\text { node positive }\end{array}$ \\
\hline Surgical bed & $4(9)$ & 3 a(6) & $0(0)$ \\
Prostate bed & $9(20)$ & $5(10)$ & $0(0)$ \\
PG + partial SV bed & $15(33)$ & $13(26)$ & $0(0)$ \\
PG + SV bed & $9(20)$ & $10(20)$ & $0(0)$ \\
Mini-pelvis & $4(9)$ & $6(12)$ & $2(4)$ \\
Whole pelvis & $1(2)$ & $13(26)$ & $54(95)$ \\
Others & $3^{\text {a) }}(7)$ & $1^{\text {b) }}(2)$ & $1^{\text {c) }}(2)$ \\
\hline
\end{tabular}

Values are presented as number (\%).

The physicians selected multiple answers.

CTV, clinical target volume; RT, radiotherapy; PG, prostate gland; SV, seminal vesicles.

${ }^{\text {a) }}$ Different depending on risk groups. ${ }^{\text {b) Gross tumor. }}{ }^{\text {c) No treat- }}$ ment.

A

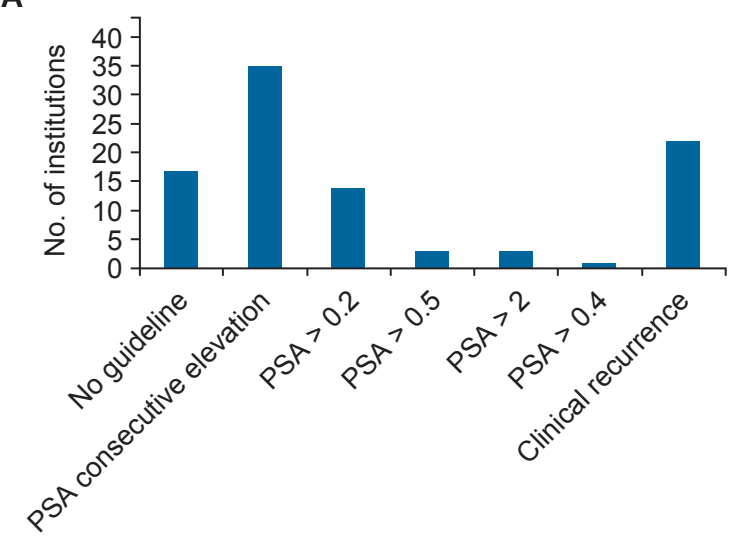

B

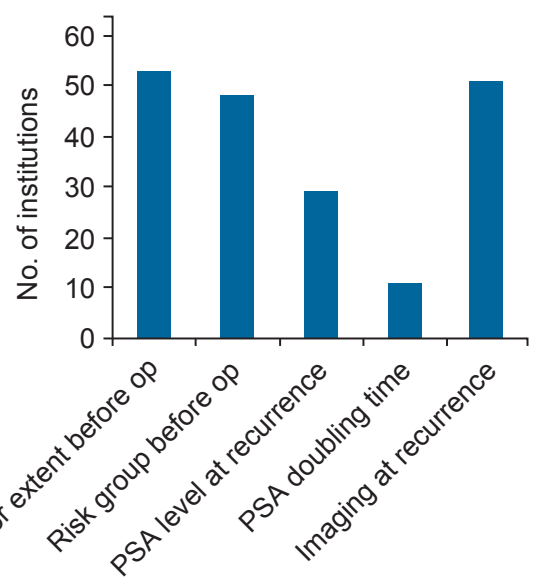

Fig. 2. (A) Indications for salvage radiotherapy in each institution. Multiple indications were allowed. (B) Factors determining clinical target volume in each institution. Multiple indications were allowed. PSA, prostate-specific antigen; op, operation.
17 hospitals (54.8\%), while HT was given 2-6 months before $\mathrm{RT}$ in 12 hospitals (38.7\%).

\section{Discussion and Conclusion}

Prostate cancer is the fourth most common cancer in men worldwide and the associated mortality is high [7]. In Korea, the prevalence of prostate cancer was much lower than that of most westernized countries, but prostate cancer has showed the highest increase in incidence rate of all the malignancies [8]. Despite this rapid increase of prostate cancer, only a few studies in treatment patterns of prostate cancer in Korean men have been reported.

In 2006, the first questionnaire based PCS in RT was conducted by 13 academic hospitals in Korea [6]. The numbers of prostate cancer patients treated with RT ranged from 60 to 150 in Seoul with 10-15 outside of Seoul in a year. Whole pelvis RT was performed in $76.9 \%$ and seminal vesicles were irradiated in $92.3 \%$ with a dose ranged from 54.0 to $73.8 \mathrm{~Gy}$. IMRT using doses higher than 70 Gy was irradiated in 53.8\% of hospitals. The number of patients was small and low dose of RT was given with relatively lower rate of IMRT usage in 2006 compared to our study. Although this study was conducted by small number of academic hospitals only, radiation dose, techniques and target volumes varied in each hospitals.

In the United States, a PCS of 9,017 prostate cancer cases diagnosed in 2004 was reported [9]. RT types and dosage utilization varied by risk group, and patients in intermediate risk groups were more likely to receive high dose RT. IMRT was used in one-third of patients to get high dose RT.

We designed this study to describe nationwide patterns of RT for prostate cancer for the first time and to use as a framework to define standard guidelines in the future. The response rate of the questionnaire survey was $72.3 \%$ among the all radiation oncologists who treat prostate cancer patients in Korea. Therefore, the results of this study could be the representative data in patterns of care for RT in curative prostate cancer.

The proportion of prostate cancer patients who received RT was 16.1\% among 2,517 curative patients in 22 hospitals in which the patient populations were reported. Considering more than 30\% of non-metastatic prostate cancer patients get $\mathrm{RT}$ in the United States, the prevalence of RT in prostate cancer was still very low in Korea $[10,11]$. Moreover, the introduction of robotic surgery in 2005 has increased the number of surgeries in prostate cancer patients and provided another surgical option for the management of patients with prostate 
cancer. In Korea, the majority of prostate cancer patients are diagnosed by urologists, therefore dominant use of surgery as curative therapy may be attributable to clinician factors [12].

IMRT was performed in 93.8\% of respondents in 2013, while IMRT was used for definitive RT in $53.8 \%$ of hospitals in 2006. RT planning and delivery systems have become more sophisticated and have moved more from conventional RT to IMRT in 2013 [13]. This change was also resulted from expansion of reimbursement for IMRT in prostate cancer by the National Health Insurance Service. Also, the use of external immobilization was increased (86\%) in 2013 comparing with the data in 2006, which showed that $76.9 \%$ of hospitals used external immobilization. In 2013, bladder control became more available for internal immobilization (70.2\% vs. $53.8 \%$ ), but rectal control including enema, gas passing, and rectal ballooning was less available than it was in 2006 (42.1\% vs. $61.5 \%)$. The most commonly prescribed radiation dose was 70 Gy (range, 60 to $81.4 \mathrm{~Gy}$ ) in 28 daily fractions for definitive RT. It was escalated to a total dose compared to the total dose of 60-78.5 Gy with 1.8 or 2.0 Gy per fraction in 2006 [6]. Recent randomized controlled trials have demonstrated a biochemical disease-free survival benefit for prostate cancer patients receiving dose escalated radiation therapy $[14,15]$. Due to the prevalence of IMRT technique, dose escalation including hypofractionation was introduced with no increase or even with a lower dose to normal critical structures, such as bowel and bladder. Different fractionation scheme was attributed to the status and patients numbers treated per day in each hospital.

The delineation of target volume including gross tumor volume (GTV), CTV, and PTV varied and whole pelvic RT was applied for different indications. Currently, there is much controversy regarding whether elective pelvic irradiation can reduce pelvic nodal recurrences and whether this technique will positively influence the treatment outcome $[16,17]$. The target volume areas were increased according to the risks but whether GTV is delineated or pelvis is included for target volume was diverse. These results may be explained by disagreement in elective pelvic irradiation in Korea. It is essential that we accurately and consistently delineate all the target volumes to conduct multi-institutional trials. Target delineation guideline relevant to each indication should be established.

Indications and timing for postoperative and salvage RT seemed to have some agreement but still diverse among hospitals. This discordance was frequently resulted from disagreement within referring physician's definition of failure or indications for postoperative RT [18]

HT was given to patients in $87.2 \%$ of hospitals. The duration of HT was not defined frequently and the timing of HT also varied. The rationale for neoadjuvant HT prior to RT is to shrink the tumor, while adjuvant HT is given to eliminate micrometastatic disease. It is unclear whether hormonal treatment combined with high dose RT can impact all the intermediate risk group patients [19-24]. Therefore, there is a need to clarify the strategy of HT for proper patients groups, duration, and timing in combination with RT for nonmetastatic prostate cancer in Korea.

One limitation of this study is that the analysis was not based on the whole prostate cancer populations in Korea. This study was conducted as a form of questionnaire survey, which could be affected by mistakes in a physician's memory or opinion. There is still need to collect data of all prostate cancer patients treated with RT. A population based study is underway by the Division for Urologic Cancer of the Korean Society for Radiation Oncology. However, the results of this study can provide useful information for assessing recent RT trends in prostate cancer.

In conclusions, this nationwide PCS suggest that variable radiation fields, techniques, and a diverse range of dose fractionation schemes are applied for prostate cancer treatment in Korea. Physicians have not reached a consensus on the practice of RT for prostate cancer treatment in Korea. Undergoing a national database work will provide better insights into defining standard guidelines for RT in Korean prostate cancer.

\section{Conflict of Interest}

No potential conflict of interest relevant to this article was reported.

\section{Acknowledgments}

We are grateful to all of the radiation oncologists who participated in this study for their efforts.

\section{References}

1. Kim IH, Chie EK, Oh DH, et al. A web-based "patterns of care study" system for clinical radiation oncology in Korea: development, launching, and characteristics. J Korean Soc Ther Radiat Oncol 2003;21:291-8.

2. Kim JH, Kim DY, Kim YH, et al. Survey on radiotherapy 
protocols for the rectal cancers among the Korean radiation oncologists in 2002 for the development of the patterns of care study of radiation therapy. J Korean Soc Ther Radiol Oncol 2003;21:44-53.

3. Hur WJ, Choi Y, Lee HS, et al. Preliminary report of the 1998-1999 patterns of care study of radiation therapy for esophageal cancer in Korea. J Korean Soc Ther Radiol Oncol 2007;25:79-92.

4. Suh CO, Shin HS, Cho JH, et al. The 1998, 1999 patterns of care study for breast irradiation after breast-conserving surgery in Korea. J Korean Soc Ther Radiol Oncol 2004;22:192-9.

5. Keum KC, Shim SJ, Lee IJ, et al. The 1998, 1999 patterns of care study for breast irradiation after mastectomy in Korea. J Korean Soc Ther Radiol Oncol 2007;25:7-15.

6. Kim JH, Kim JS, Ha SW, et al. A patterns of care study of the various radiation therapies for prostate cancer among Korean radiation oncologists in 2006. J Korean Soc Ther Radiol Oncol 2008;26:96-103.

7. Siegel R, Ma J, Zou Z, Jemal A. Cancer statistics, 2014. CA Cancer J Clin 2014;64:9-29.

8. Jung KW, Won YJ, Kong HJ, et al. Cancer statistics in Korea: incidence, mortality, survival, and prevalence in 2012. Cancer Res Treat 2015;47:127-41.

9. Wang D, Ho A, Hamilton AS, et al. Type and dose of radiotherapy used for initial treatment of non-metastatic prostate cancer. Radiat Oncol 2014;9:47.

10. Aneja S, Smith BD, Gross CP, et al. Geographic analysis of the radiation oncology workforce. Int J Radiat Oncol Biol Phys 2012;82:1723-9.

11. Mahmood U, Levy LB, Nguyen $P L$, Lee $A K$, Kuban DA, Hoffman KE. Current clinical presentation and treatment of localized prostate cancer in the United States. J Urol 2014;192:1650-6.

12. Park J, Suh B, Shin DW, Hong JH, Ahn H. Changing patterns of primary treatment in Korean men with prostate cancer over 10 years: a nationwide population based study. Cancer Res Treat 2016;48:899-906.

13. Wang-Chesebro A, Xia P, Coleman J, Akazawa C, Roach M 3rd. Intensity-modulated radiotherapy improves lymph node coverage and dose to critical structures compared with threedimensional conformal radiation therapy in clinically localized prostate cancer. Int J Radiat Oncol Biol Phys 2006;66:654-62.

14. Beckendorf $V$, Guerif $S$, Le Prise $E$, et al. 70 Gy versus $80 \mathrm{~Gy}$ in localized prostate cancer: 5 -year results of GETUG 06 randomized trial. Int J Radiat Oncol Biol Phys 2011;80:105663.
15. Zietman AL, DeSilvio ML, Slater JD, et al. Comparison of conventional-dose vs high-dose conformal radiation therapy in clinically localized adenocarcinoma of the prostate: a randomized controlled trial. JAMA 2005;294:1233-9.

16. Lawton CA, DeSilvio M, Roach M 3rd, et al. An update of the phase III trial comparing whole pelvic to prostate only radiotherapy and neoadjuvant to adjuvant total androgen suppression: updated analysis of RTOG 94-13, with emphasis on unexpected hormone/radiation interactions. Int J Radiat Oncol Biol Phys 2007;69:646-55.

17. Pommier $P$, Chabaud $S$, Lagrange $J L$, et al. Is there a role for pelvic irradiation in localized prostate adenocarcinoma? Preliminary results of GETUG-01. J Clin Oncol 2007;25:536673.

18. Bolla $M$, van Poppel $H$, Collette $L$, et al. Postoperative radiotherapy after radical prostatectomy: a randomized controlled trial (EORTC trial 22911). Lancet 2005;366:572-8.

19. Denham JW, Joseph D, Lamb DS, et al. Short-term androgen suppression and radiotherapy versus intermediate-term androgen suppression and radiotherapy, with or without zoledronic acid, in men with locally advanced prostate cancer (TROG 03.04 RADAR): an open-label, randomised, phase 3 factorial trial. Lancet Oncol 2014;15:1076-89.

20. Pilepich MV, Winter $K_{1}$ John MJ, et al. Phase III radiation therapy oncology group (RTOG) trial 86-10 of androgen deprivation adjuvant to definitive radiotherapy in locally advanced carcinoma of the prostate. Int J Radiat Oncol Biol Phys 2001;50:1243-52.

21. Denham JW, Steigler A, Lamb DS, et al. Short-term androgen deprivation and radiotherapy for locally advanced prostate cancer: results from the Trans-Tasman Radiation Oncology Group 96.01 randomised controlled trial. Lancet Oncol 2005;6:841-50.

22. Bolla M, de Reijke TM, Van Tienhoven $G$, et al. Duration of androgen suppression in the treatment of prostate cancer. $\mathrm{N}$ Engl J Med 2009;360:2516-27.

23. Horwitz EM, Bae K, Hanks GE, et al. Ten-year follow-up of radiation therapy oncology group protocol 92-02: a phase III trial of the duration of elective androgen deprivation in locally advanced prostate cancer. J Clin Oncol 2008;26:2497-504.

24. Denham JW, Steigler A, Lamb DS, et al. Short-term neoadjuvant androgen deprivation and radiotherapy for locally advanced prostate cancer: 10-year data from the TROG 96.01 randomised trial. Lancet Oncol 2011;12:451-9. 